\title{
Evolution of the scholarly mega-journal, 2006-2017
}

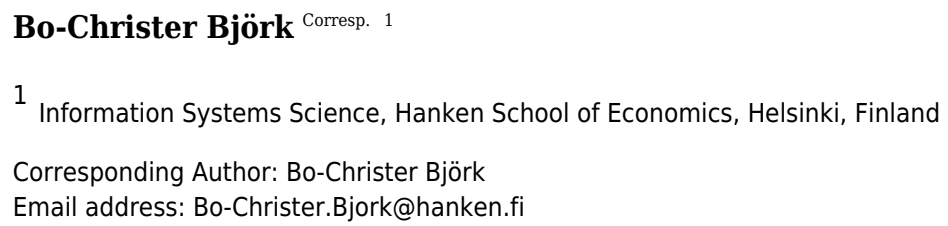

Mega-journals are a new kind of scholarly journals, made possible by electronic publishing. They are open access (OA) and funded by charges, which authors pay for the publishing services. What distinguishes mega-journals from other OA journals is in particular a peer review focusing only on scientific trustworthiness. The journals can easily publish thousands of articles per year and there is no need to filter articles due to restricted slots in the publishing schedule. This study updates some earlier longitudinal studies of the evolution of mega-journals and their publication volumes. After very rapid growth in 20102013, the increase in overall article volumes has slowed down. Mega-journals are also increasingly dependent for sustained growth on Chinese authors, who now contribute $25 \%$ of all articles in such journals. There has also been an internal shift in market shares. PLOS ONE, which totally dominated mega-journal publishing in the early years, currently publishes around one third of all articles. Scientific Reports has grown rapidly since 2014 and is now the biggest journal. 
1

2 Evolution of the scholarly mega-journal, 2006-2017

3

4 Bo-Christer Björk, Information Systems Science, Hanken School of Economics,

5 Helsinki, Finland

6 Bo-Christer.Bjork@hanken.fi

7

8

\section{Abstract}

Mega-journals are a new kind of scholarly journals, made possible by electronic publishing. They are open access $(\mathrm{OA})$ and funded by charges, which authors pay for the publishing services. What distinguishes mega-journals from other OA journals is in particular a peer review focusing only on scientific trustworthiness. The journals can easily publish thousands of articles per year and there is no need to filter articles due to restricted slots in the publishing schedule. This study updates some earlier longitudinal studies of the evolution of mega-journals and their publication volumes. After very rapid growth in 20102013, the increase in overall article volumes has slowed down. Mega-journals are also increasingly dependent for sustained growth on Chinese authors, who now contribute 25 $\%$ of all articles in such journals. There has also been an internal shift in market shares. PLOS ONE, which totally dominated mega-journal publishing in the early years, currently publishes around one third of all articles. Scientific Reports has grown rapidly since 2014 and is now the biggest journal. 


\section{Introduction}

Electronic dissemination on the web has long been envisioned as a "game changer" for the publishing of scholarly peer reviewed journals. Open access publishing, in which readers no longer pay for access content, has become possible due to this (Suber, 2012). Nevertheless, leading mainstream publishers have been slow in adapting OA, simply because the subscription model is still very lucrative (Björk, 2017a). The big change in their business model has been from paper to electronic delivery, and the bundling of journals into huge e-licenses.

The leading publishers (commercial, society and university press) have consequently been quite cautious in starting new $\mathrm{OA}$ journals or converting existing journals to open access funded by author-side payments. They have instead partially opened around 10,000 journals in a hybrid form, in which authors can pay to make their articles open in otherwise closed subscription journals (Laakso \& Björk, 2016).

Electronic web delivery has also made possible experiments with new types of peer review, for instance open peer review, in which the manuscripts have been posted on the web and readers provide reviews. One type of review which web publishing indirectly has facilitated is review based only on scientific soundness, not on the perceived importance of the findings. The reason is that electronic only $\mathrm{OA}$ journals no longer need to restrict the number of articles yearly published, but can easily scale up according to the number of submissions.

Using such review methods several publishers have started so-called mega-journals, in the wake of the phenomenal success of the pioneering PLOS ONE journal. Over the last ten years the total article output of such journals has rapidly grown, and nowadays constitutes a significant share of all output in $\mathrm{OA}$ journals.

A number of authors have proposed slightly varying definitions of what constitutes a megajournal (Norman, 2012), (Spezi et al, 2017). The definition of a mega-journal used in this study is the same as in (Björk, 2015). A mega-journal has to fulfil five primary criteria;

- A big publishing volume or aiming at it

- Peer review by scientific soundness only

- Broad subject area

- Full open access

- Funded by authors paying publishing fees

In addition, a mega-journal should fulfil several (but not necessarily all) of a number of secondary criteria. These include:

- Rapid publication

- Moderate author fee

- High prestige publisher 
65 For the full list see (Björk, 2015).

66

67

68

The aim of this study was to provide new updated data on the longitudinal evolution of mega-journal output, and to compare that with the article volumes of related phenomena such as articles in full OA journals and hybrid OA journals.

\section{Earlier research}

72

There have been relatively few empirical studies of mega-journals. In addition, there have been a number of interesting newsletter and blog items, which to some extent have reported data, but which also express opinions about the phenomenon. In addition to the more scholarly studies, advocates for $\mathrm{OA}$ and sceptics have debated the merits and dangers of mega-journals. Titles such as: "Open Access Megajournals - have they changed everything" (Binfield, 2013), " and "Mega-journals: the future, as stepping stone to it or a leap into the abyss?" (Pinfield, 2016) describe the discourse pretty well.

Academic studies concerning mega-journals have covered a number of aspects. Topics which have been covered in the earlier literature include:

- Definition of a mega-journal, features, lists of journals (Norman, 2012), Björk, 2015), (Spezi et al, 2017).

- Bibliometric studies of citations etc. (Björk \& Catani, 2016), (Wakeling et al, 2016).

- Author surveys, factors affecting journal choice etc. (Solomon, 2013).

- Case studies of individual journals (Wilson \&Humphrey, 2017), (Wakeling et al, 2017).

Spezi et al (2017) provides an excellent review of the literature to date, and the reader is referred to that article for a more in-depth discussion.

There have been a handful of studies and blog-items that in particular have included data on the article volume development of mega-journals (Binfield, 2013), (Björk, 2015), (Spezi et al, 2017). The range of included journals varied somewhat but since all tend to have included the leading journals PLOS ONE and Scientific Reports, they are roughly comparable. All these show a very rapid growth period from 2010 , which seems to have started levelling out in 2013-2015.

\section{Methods}

The basis for the list of mega-journals studied were the 14 journals which had been identified in the earlier study (Björk, 2015). Springer Plus was included, despite the fact that it has ceased publishing from the start of 2017. In addition, five additional journals were added. Medicine, F1000 research, and BMC research notes had been included in the 
102 previous study of Spezi et al. (2017). Heliyon started publishing only recently. The Cogent

103 series of 15 mega-journals (Cogent Engineering, Cogent Social Sciences etc.) were also

104 added and considered as one journal.

105 The publication volumes for the journals were checked 15-16.1.2018 using Scopus for all

106 the journals included in that index, using the advanced search function which allows

107 searching articles in a particular journal. Only articles were included and all other types of

108 indexed items (reviews, errata, retractions) were excluded. In the case of the Cogent series

109 of journals and the Journal of Engineering articles were hand counted from the websites.

110 The share of Chinese authors in the journals was obtained using the Scopus numerical

111 breakdown of country affiliation of the authors. Thus the count is based on where the 112 author is working, not directly on nationality. What Scopus counts are articles with at least

113 one author from the country in question. Since many articles have more than one author, 114 the sum of the country affiliations will be higher than the number of articles (had only the

115 corresponding authors been counted the sums would be equal). This is not a problem if the 116 longitudinal changes in shares are studied or in comparisons between countries or with 117 other disciplines. The same method has been used in the earlier study by Wakeling et al. 118 (2016).

\section{Results}

\section{Longitudinal development}

121

122

123

124

125

126

127

128

129

130

131

132

133

134

135

136

137

138

139

140

141

142

From a longitudinal perspective the evolution of mega-journals can be split into a number of major phases. During the first phase PLOS ONE was the one and only of its kind and grew from 138 articles in 2006 to 6,864 in 2009. When its success started to be apparent several other established mainstream publishers launched their own mega-journals. Nine of the journals in this study were launched in either 2011 or 2012 and the period up to 2013 saw a rapid growth in combined output. From 2015 onwards the major developments have been that Scientific Reports has caught up with and surpassed PLOS ONE in article numbers, and that many of the middle tier journals have consolidated their position. The overall development is shown in table 1.

Table 1. Development of article volumes in mega-journals 2010-2017.

The journals can be grouped into four groups, each with its own characteristics. The first one consists of PLOS ONE and Scientific Reports, which each contribute around one third of all mega-journal articles. The second group consists of the single subscription journal Medicine, which converted to an OA mega-journal in 2014. The article volume prior to conversion in 2012-2013 is shown in parenthesis, and demonstrates a staggering hundredfold growth in just a couple of years.

The third group contains six journals with between 1,000 - 2,000 articles per annum. Of these three are from highly reputable society publishers with portfolios of several journals (BMJ, AIP and IEEE). PeerJ is a start-up with no prior publisher brand name to leverage. Springer Plus also belonged to this category before the journal stopped publishing in 2017 . 
143 Cogent has preferred to split, what in this study is regarded as one mega-journal, into 15 144 distinct journals together covering all sciences. The journals in this middle tier contribute $14514 \%$ of all articles.

146 The fourth and last group includes the remaining 10 journals, with predicted journal 147 volumes of clearly less than 1,000 in 2017. Summed up they only publish $5 \%$ of all articles. 148 In this group there is one journal, which is concentrating only the social sciences and 149 humanities; Sage Open. Only two of the journals in the group have so far JCR impact factors.

150

151

152

153

154

155

156

157

158

159

160

161

162

163

164

165

166

167

168

169

170

171

172

173

174

175

176

177

178

179

180

181

182

183

\section{Rising share of Chinese authors}

There has been a shift in the origin of authors who publish in mega-journals. Of particular interest is the high proportion in some of the biggest journals of authors affiliated with Chinese universities or institutes. Already Wakeling et al (2016) in their bibliometric analysis noted a Chinese share of around $40 \%$ in both Scientific Reports, AIP Advances and Medicine. For this study Chinese author shares for the same journals and some additional journals were estimated from 2013 to 2017 using the Scopus index search facility. The results are shown in table 2 . As a comparison point the overall percentage of China based authors of all Scopus articles was $16 \%$ in 2013 and $20 \%$ in 2017.

Table 2. Share of authors with affiliation in China in mega-journal publication volumes.

The distribution over the journals is highly skewed, two journals having more than half Chinese authors, and five over $30 \%$. Overall the share has risen but seems to have stabilised around $25 \%$.

\section{Discussion}

The article output of mega-journals should be seen in context, for instance as part of the publications from all credible peer reviewed journals (so-called predatory OA journals excluded). A good tool for measuring this is the Scopus index which currently indexes around 20,700 mostly English language journals, including 17 of the 19 journals in this study.

Between 2010 and 2016 the overall number of articles indexed in Scopus grew by $28 \%$, to around 2,170,000. In a separate on-going study together with Mikael Laakso, we have estimated that in 2010 the share of OA articles of all Scopus articles was 10,3\% and grew to 19,4 by 2016 . The by far biggest growth rates in this period were for mega-journals $(0,4$ to 2,6 \% Scopus share) and articles in hybrid OA journals $(0,6$ to 2,0$)$. The numbers for hybrid journals are from a recent separate study (Björk, 2017b). In 2010 almost every second OA article was still published in a journal not charging authors $(81,000$ vs 93,000 in APC-charging) but by 2016 charging the authors had started dominating the picture (129,000 free vs 293,000 for which APCs were paid).

This study is a straightforward empirical study using robust data available from highquality indexing services. No sampling has been required. It is easily replicable and can also be renewed at a later stage to study subsequent developments. The results for the earlier 
184 years are well in line with the results from research reported in the "earlier research"

185 section. The minor differences can be explained by slightly different lists of included

186 journals and using Scopus vs counting articles from journal websites. A key challenge is

187 obviously also in future studies to identify new Mega-journals as such are started up or

188 converted from subscription journals.

189 A very challenging future research topic is what the effects of the "scientific soundness"

190 only review criterion has on the internal citation patterns of articles in mega-journals vs.

191 traditional journals (Björk \& Catani, 2016), (Wakeling et al, 2016).

192

\section{Conclusions}

194

All in all, the developments in article numbers indicate that mega-journals have found a

196 place in scholarly publishing. From a business perspective they complement well the journal portfolios of major commercial and society publishers, and thrive in symbiosis with more selective journals, for instance via rejected submissions being redirected to them via so-called cascading reviews (Spezi et al, 2017). Mega-journals will not revolutionize the industry and the way mainstream peer review works, but they cater to the needs of

201

202 particular groups of authors in providing rapid publication, better predictability of getting a submission accepted and reasonable brand recognition in publication lists.

203

\section{References:}

205

Binfield P. 2013. Open Access Megajournals - have they changed everything? Blog post, Creative Commons Aotearoa New Zealand, http://creativecommons.org.nz/2013/10/open-access-megajournals-have-theychanged-everything/

Björk B-C. 2015. Have the "mega-journals" reached the limits to growth? PeerJ 3: e981. doi: 10.7717 peerj.981

Björk B-C. 2017a. Scholarly journal publishing in transition- from restricted to open access, Electronic Markets, The International Journal on Networked Business 27: 101-109. doi: 10.1007/s12525-017-0249-2

Björk B-C. 2017b. Growth of hybrid open access, 2009-2016. PeerJ 5:e3878. doi; 10.7717/peerj.3878

Björk B-C, Catani P. 2016. Peer review in megajournals compared with traditional scholarly journals: Does it make a difference? Learned Publishing 29: Issue 1, 9-12. doi: 10.1002/leap.1007

Laakso M, Björk B-C. 2016. Hybrid Open Access - a longitudinal study , Journal of Informetrics 10: 919-932 . doi: 10.1016/j.joi.2016.08.002

218 Norman, F. 2012. Megajournals, blog post, Occam's typewriter. available at http://occamstypewriter.org/ 219 trading-knowledge/2012/07/09/megajournals/

Pinfield S. 2016. Mega-journals: the future, as stepping stone to it or a leap into the abyss? Times Higher Education, Oct 13, 2016. https://www.timeshighereducation.com/blog/mega-journals-future-steppingstone-it-or-leap-abyss

223 Solomon D. 2013. A survey of authors publishing in four megajournals. PeerJ 2: e365. doi: 10.7717/peerj.365 
224 Spezi V, Wakeling S, Pinfield S, Creaser C, Fry J, Willett P. 2017. Open-access mega-journals: The future of 225 scholarly communication or academic dumping ground? A review. Journal of Documentation 73: 263-283. doi: 226 https://doi.org/10.1108/JD-06-2016-0082

227 Suber P. 2012. Open Access, MIT Press. Cambridge, Massacusetts, 230p, OA version available at 228 https://mitpress.mit.edu/sites/default/files/9780262517638_Open_Access_PDF_Version.pdf

229 Wakeling S, Willett P, Creaser C, Fry J, Pinfield, S, Spezi, V. 2016. Open-Access Mega-Journals: A 230 Bibliometric Profile, PLOS ONE 11: e0165359. doi:10.1371/journal.pone.0165359.s001

231 Wakeling S, Willett P, Creaser C, Fry J, Pinfield S, Spezi V. 2017. Transitioning from a Conventional to a 232 'Mega' Journal: A Bibliometric Case Study of the Journal Medicine. Publications 5: e7. doi:

233 10.3390/publications5020007

234 Wilson E, Humphrey J. 2017. Successfully transitioning the world's largest chemistry subscription journal to 235 a gold open access publication. Insights 30: 38-46. doi: http://doi.org/10.1629/uksg.343 


\section{Table 1 (on next page)}

Development of article volumes in mega-journals 2006-2017. 
1 Table 1

2

\begin{tabular}{|c|c|c|c|c|c|c|c|c|}
\hline \multirow[t]{2}{*}{ JOURNAL: } & \multicolumn{8}{|c|}{ Number of published research articles } \\
\hline & 2010 & 2011 & 2012 & 2013 & 2014 & 2015 & 2016 & 2017 \\
\hline \multicolumn{9}{|l|}{ "Big Two": } \\
\hline Scientific Reports & & 208 & 819 & 2498 & 3940 & 10707 & 20358 & 24077 \\
\hline PLOS ONE & 6864 & 13701 & 23426 & 31404 & 30398 & 27858 & 21770 & 20098 \\
\hline \multicolumn{9}{|l|}{ Converted journals: } \\
\hline Medicine & & & $(22)$ & $(29)$ & 296 & 1814 & 2844 & 2761 \\
\hline \multicolumn{9}{|l|}{ Middle tier: } \\
\hline Springer Plus & & & 77 & 666 & 743 & 881 & 2011 & 0 \\
\hline IEEE Access & & & & 62 & 118 & 230 & 758 & 2070 \\
\hline BMJ Open & & 98 & 625 & 894 & 1059 & 1292 & 1735 & 1683 \\
\hline Cogent Series & & & & & 110 & 516 & 1298 & 1432 \\
\hline AIP Advances & & 251 & 373 & 396 & 558 & 930 & 1240 & 1395 \\
\hline PeerJ & & & & 229 & 474 & 826 & 1309 & 1367 \\
\hline \multicolumn{9}{|l|}{ Smaller journals: } \\
\hline BMC Research Notes & 343 & 544 & 673 & 532 & 958 & 870 & 526 & 739 \\
\hline Royal Society Open Science & & & & & 50 & 246 & 414 & 648 \\
\hline G3 & & 63 & 167 & 249 & 418 & 323 & 285 & 352 \\
\hline F1000 Research & & & 42 & 204 & 269 & 200 & 421 & 325 \\
\hline Sage Open & & 46 & 116 & 222 & 326 & 288 & 367 & 304 \\
\hline Heliyon & & & & & & 29 & 156 & 249 \\
\hline Biology Open & & & 140 & 160 & 137 & 183 & 217 & 218 \\
\hline FEBS Open Bio & & 4 & 52 & 78 & 121 & 110 & 118 & 170 \\
\hline Journal of Engineering & & & & 20 & 102 & 80 & 69 & 92 \\
\hline Elementa, Science of the Antropocene & & & & 12 & 12 & 39 & 52 & 27 \\
\hline ALL MEGAJOURNALS & 7207 & 14915 & 26510 & 37626 & 40089 & 47422 & 55948 & 58007 \\
\hline Big two (2) & 6864 & 13909 & 24245 & 33902 & 34338 & 38565 & 42128 & 44175 \\
\hline Converted (1) & & & & & 296 & 1814 & 2844 & 2761 \\
\hline Middle tier (6) & & 349 & 1075 & 2247 & 3062 & 4675 & 8351 & 7947 \\
\hline Smaller journals (10) & & 685 & 1269 & 1472 & 2241 & 2308 & 2781 & 3158 \\
\hline
\end{tabular}




\section{Table 2 (on next page)}

Share of authors with affiliation in China in mega-journal publication volumes. 
1 Table 2

2

\begin{tabular}{|l|l|l|l|l|l|l|}
\hline JOURNAL: & \multicolumn{6}{l}{$\begin{array}{l}\text { Share of authors with an } \\
\text { affiliation in China (\%) }\end{array}$} \\
\hline & $\mathbf{2 0 1 3}$ & $\mathbf{2 0 1 4}$ & $\mathbf{2 0 1 5}$ & $\mathbf{2 0 1 6}$ & $\mathbf{2 0 1 7}$ \\
\hline \multicolumn{5}{|l|}{} \\
\hline & 6 & 14 & 24 & 47 & 55 \\
\hline IEEE Access & 0 & 28 & 37 & 39 & 54 \\
\hline Medicine & 32 & 42 & 40 & 40 & 40 \\
\hline AIP Advances & 29 & 39 & 39 & 37 & 31 \\
\hline Scientific Reports & 6 & 6 & 19 & 26 & 30 \\
\hline FEBS Open Bio & 3 & 1 & 6 & 15 & 20 \\
\hline Biology Open & 19 & 20 & 19 & 16 & 16 \\
\hline PLOS ONE & 2 & 4 & 7 & 15 & 16 \\
\hline PeerJ & & 2 & 3 & 3 & 8 \\
\hline Royal Society Open Science & 6 & 5 & 7 & 11 & 7 \\
\hline G3 & 4 & 7 & 7 & 7 & 7 \\
\hline BMJ Open & & & 14 & 9 & 5 \\
\hline Heliyon & 2 & 2 & 1 & 2 & 2 \\
\hline BMC Research Notes & 2 & 2 & 2 & 2 & 2 \\
\hline F1000 Research & 0 & 1 & 1 & 1 & 1 \\
\hline SAGE Open & 8 & 0 & 0 & 4 & 0 \\
\hline Elementa, Science of the Antropocene & & & & & \\
\hline & $\mathbf{1 8}$ & $\mathbf{2 1}$ & $\mathbf{2 3}$ & $\mathbf{2 5}$ & $\mathbf{2 5}$ \\
\hline IN ALL JOURNALS & & &
\end{tabular}

3

4 